\title{
PLANEACIÓN ESTRATÉGICA EN ORGANIZACIONES DE LA SOCIEDAD CIVIL. UN BREVE ANÁLISIS PARA EL ESTADO DE GUANAJUATO
}

\author{
D.D C. CALDERA ${ }^{*}$, M.A. ORTEGA y M.E. SÁNCHEZ \\ Universidad de Guanajuato, México \\ dccaldera@ugto.mx*
}

Submetido 28/06/2016 - Aceito 25/05/2017

DOI: $10.15628 /$ holos. 2017.4762

\section{RESUMEN}

Las Organizaciones de la Sociedad Civil (OSC) representan una gama de oportunidades para el diseño e implementación de proyectos que impulsen el desarrollo humano sustentable, y por ende, son actores estratégicos para lograr la Responsabilidad Social.

Sin embargo en México el número de OSC es reducido comparado con otros países, además de que son organizaciones que apenas están siendo consideradas por la sociedad en general.

Este trabajo muestra resultados de un Diagnóstico de OSC en Guanajuato, tomando como eje central a la Planeación Estratégica, ya que es bien sabido que en el proceso administrativo es vital la planeación, que consiste en decidir anticipadamente qué hacer, cuándo y cómo hacerlo, lo cual ayuda a prever un rumbo y orientar el futuro deseado.

Se parte de que una adecuada planeación permitirá a las OSC fijar objetivos precisos y alcanzables, así como proveerse de información para lograr un profundo autoconocimiento. Así las OSC tendrán claros sus puntos fuertes y débiles, para poder desarrollarse e interactuar exitosamente tanto de manera interna como con su exterior y con ello será posible tener un mayor impacto.

PALABRAS CLAVE: Planeación, Estrategia, Organizaciones, Sociedad Civil.

\section{STRATEGIC PLANNING IN CIVIL SOCIETY ORGANIZATIONS. A BRIEF ANALYSIS TO GUANAJUATO, MÉXICO}

\begin{abstract}
The Civil Society Organizations (CSO) represent a range of opportunities to design and implement projects that promote sustainable human development, and therefore they become strategic actors to achieve social responsibility.

However, in Mexico, the number of CSO is low compared with other countries, and they are just being considered by society in general.

This paper shows the results of a diagnosis of CSO in Guanajuato, taking as central axis Strategic Planning, because is well known that in the administrative process
\end{abstract}

is vital planning, which is to decide in advance what to do, when and how to do it, that helps to provide a direction and guide the desired future.

It is assumed that proper planning will enable CSO to set precise and achievable objectives as well as information provided to achieve a deep self-knowledge. In this way, CSO will clear their strengths and weaknesses, to develop and successfully interact both internally and with the outside and then it will be possible have a major social impact.

KEYWORDS: Planning, Strategy, Civil Society, Organization. 


\section{INTRODUCCIÓN}

Las Organizaciones de la Sociedad Civil (OSC) representan una gama de posiciones frente a la problemática social y a las políticas que se ponen en práctica para atenderla, ofreciendo una gran oportunidad para el diseño e implementación de proyectos que impulsen el desarrollo humano sustentable.

De esta manera se logra la mejora de las condiciones y calidad de vida de grupos vulnerables. Lamentablemente en México, el número de OSC es reducido comparándose con otros países, además de que son organizaciones relativamente recientes y su marco jurídico también se encuentra en ciernes.

Si analizamos contextos locales determinados, la problemática se agrava, ya que existen entidades de la República Mexicana con escasa existencia de OSC, además de que se ha identificado en diversas investigaciones, que dichas organizaciones tienen diferentes áreas de oportunidad, destacando principalmente la necesidad de profesionalización para poder fortalecerse, esto con la finalidad de que su impacto social sea mayor y que su labor sea sustentable en el largo plazo.

El contexto en el cual se desenvuelve esta investigación es el estado de Guanajuato, el cual se encuentra situado en la Región Bajío de la República Mexicana, y se ubica en el lugar seis de treinta y dos estados en densidad poblacional con 5 millones 486 mil 372 habitantes (INEGI, 2015), y respecto al número de OSC activas encontramos tan sólo 544.

Como puede apreciarse, simplemente en términos de densidad asociativa nos encontramos ante una gran área de oportunidad, motivo por el cual se realizó esta investigación.

El presente trabajo muestra algunos resultados de un Diagnóstico de OSC establecidas en el estado de Guanajuato. Dicho diagnóstico se realizó a través de una encuesta estructurada en cinco ejes temáticos: a) Estructura y Perfil Organizacional; b) Factor Humano y Profesionalización; c) Planeación Estratégica; d) Procuración de Fondos, Transparencia y Rendición de Cuentas; y e) Infraestructura. Para este trabajo sólo se presentarán resultados los del eje temático de Planeación Estratégica.

Es bien sabido que en el proceso administrativo es vital la planeación, la cual consiste en decidir anticipadamente qué hacer, cuándo y cómo hacerlo, lo cual ayuda a prever un rumbo y orientar el futuro deseado. Siguiendo a Bryson (2011), la planeación estratégica hoy es más importante que nunca, ya que nos encontramos ante un ambiente turbulento e incierto, en el cual tener una guía u orientación es imprescindible.

Se parte del hecho de que una adecuada planeación permitirá a las OSC fijar objetivos precisos y alcanzables, así como proveerse de información para lograr un profundo autoconocimiento. Así las OSC tendrán claros sus puntos fuertes y débiles, para poder desarrollarse e interactuar exitosamente tanto de manera interna como con su exterior.

\section{REVISIÓN BIBLIOGRÁFICA}

\section{ORGANIZACIONES DE LA SOCIEDAD CIVIL EN MÉXICO Y EN GUANAJUATO}

En México utilizamos el término de organizaciones de la sociedad civil (OSC) para referirnos a lo que internacionalmente se conoce como organizaciones no gubernamentales $u$ organizaciones sin fines de lucro. OSC es el término jurídico con las cuales son reconocidas estas organizaciones de acuerdo a la Ley Federal de Fomento, la cual entró en vigor en el año 2004.

Siguiendo a Caldera (2013, p. 13), en esta Ley, son definidas como "aquellas agrupaciones u organizaciones legalmente constituidas, sin fines de lucro, sin proselitismo partidista ni político 
electoral, sindical o religioso, que desempeñen actividades como asistencia social; apoyo a la alimentación popular; actividades cívicas; asistencia jurídica; apoyo al desarrollo de los pueblos y comunidades indígenas; promoción de la equidad de género; etc."

También conocidas como organizaciones del tercer sector al no pertenecer ni al Estado ni al Mercado, estas organizaciones se han abierto camino en la defensa de derechos humanos y la búsqueda de la justicia, tarea nada sencilla en un país con altos índices de marginación, pobreza e inseguridad como lo es México, con una población total de 119 millones 530 mil 753 habitantes (INEGI, 2015) y tan sólo 20 mil 171 OSC activas (RFOSC, 2016) formalmente registradas en el padrón oficial en todo el país ${ }^{1}$.

Como se mencionó en el apartado introductorio de esta investigación, tan sólo en términos de densidad asociativa nos encontramos ante una gran área de oportunidad, motivo por el cual se realizó esta investigación para ver de qué manera es posible que las organizaciones que existen en una entidad federativa cuenten con herramientas administrativas que les permitan tener un impacto mayor en sus actividades cotidianas.

Las OSC buscan generalmente la resolución de problemas de índole social. La pobreza es una de las problemáticas más frecuentes que trae consigo otras carencias. De acuerdo con el Consejo Nacional para la Evaluación de la Política para el Desarrollo Social (CONEVAL), una persona se encuentra en situación de pobreza cuando tiene al menos una carencia social (en los seis indicadores de rezago educativo, acceso a servicios de salud, acceso a la seguridad social, calidad y espacios de la vivienda, servicios básicos en la vivienda y acceso a la alimentación) y su ingreso es insuficiente para adquirir los bienes y servicios que requiere para satisfacer sus necesidades alimentarias y no alimentarias. Bajo esta premisa, en el estado de Guanajuato, los índices de pobreza no son nada alentadores, ya que por ejemplo en 2014, fecha de la última evaluación se encontró que $46.6 \%$ de la población se encontraba en situación de pobreza, y de este porcentaje el 5.5\% en pobreza extrema (CONEVAL, 2014).

Aunado a lo anterior, otros indicadores de carencias sociales muestran que existe un $21 \%$ de rezago educativo, $15.4 \%$ de la población carece de servicios de salud, $57.9 \%$ no cuenta con acceso a seguridad social; $9.8 \%$ no tiene acceso a calidad y espacios en la vivienda; $14.9 \%$ no tiene los servicios básicos en su vivienda y $22.9 \%$ carece de acceso a la alimentación (CONEVAL, 2014).

Como puede observarse, es tarea apremiante la búsqueda de alternativas para que estos indicadores cambien. Así, las OSC son una opción en la búsqueda de mejores condiciones de vida para la población en general, sin embargo para que resulte viable, es necesario que se profesionalicen. A continuación se abordará el tema de la planeación, ya que consideramos que es esencial en el trabajo de las OSC.

\section{LA IMPORTANCIA DE LA PLANEACIÓN EN LAS ORGANIZACIONES}

Las organizaciones, independientemente del ramo o su área concerniente, debieran capacitarse, profesionalizarse y fortalecerse para lograr un impacto social mayor y que su labor sea sustentable en el largo plazo.

Por lo anterior se realza la importancia que tiene una adecuada planeación, la cual permitirá a las OSC alcanzar sus metas a corto y largo plazo.

Henri Fayol, destacado teórico en la disciplina administrativa centró el proceso administrativo en 4 funciones básicas: planear, organizar, dirigir y controlar. Así, la primera etapa

\footnotetext{
${ }^{1}$ En México, existe el Registro Federal de OSC, que es administrado por la Secretaría de Desarrollo Social a través del Instituto Nacional de Desarrollo Social (Indesol).
} 
del proceso administrativo está constituida por la previsión, la cual consiste en planear. Planear es decidir anticipadamente qué hacer, cuándo y cómo hacerlo, lo cual ayuda a prever un rumbo y orientar el futuro deseado.

Para lograr una acertada planeación en la organización, se deben considerar diversos aspectos, tales como: determinar una clara misión y visión de la organización, desarrollar un amplio y certero análisis estratégico (análisis de fortalezas, oportunidades, debilidades y amenazas FODA), diseño de reglamentos, manuales y planes, así como dar seguimiento a los programas implementados y realizar alianzas u obtener apoyo de diversos organismos (Fred, 2008; Thompson \& Strickland, 2004; Mintzberg, Quinn \& Voyer, 1993; Hill \& Jones, 2009).

Hablaremos de cada aspecto primordial de manera más amplia, empezando con la importancia de que una adecuada planeación debe plasmar por escrito la misión y visión de la organización, ya que estos aspectos son parte de la planeación estratégica.

La misión muestra una declaración positiva de lo que hacemos, quiénes somos, qué buscamos, para qué lo hacemos, y para quiénes trabajamos; la visión resume en un enunciado, la imagen prospectiva de lo que la asociación quiere llegar a ser en un futuro (Mintzberg, Quinn \& Voyer, 1993; Hill \& Jones, 2009).

La misión es parte del autoconocimiento, nos permite definir claramente nuestras funciones $y$ en qué entorno nos desarrollaremos. Cuando nos conocemos realmente como organización, podremos tener una visión clara de nuestro futuro organizacional.

Aunado a lo anterior, debemos tener claro que el éxito de una organización, en gran parte se debe a una buena administración con reglas claras y explícitas respecto a los roles que cada miembro debe cumplir. Generalmente en las organizaciones esto se realiza plasmado en reglamentos, manuales y planes, a los cuales debe darse seguimiento $y$, en un horizonte determinado de tiempo, han de evaluarse para verificar su efectividad (Hitt, Ireland \& Hoskisson, 2008).

Así bien, resulta importante identificar de qué manera las OSC plasman sus reglas y los roles de los individuos que integran la organización, lo cual puede realizarse a través de la sistematización de la experiencia, ya que de esta manera las organizaciones serán capaces de reconstruir y ordenar sus actividades, con lo cual pueden descomponer y analizar los procesos vividos, y a su vez pueden encontrar áreas de oportunidad para mejorarlas.

Otro aspecto muy importante que es necesario considerar en la planeación para las OSC, es la realización del análisis FODA, definido de manera más amplia como una herramienta para identificar en qué condiciones se encuentra la organización en un contexto determinado, por lo cual muestra aquellos aspectos que son capaces de generar impactos negativos y positivos para la realización de las actividades de la organización. Este análisis puede realizarse de manera participativa con los miembros de la organización.

Siguiendo a Hill \& Jones (2009), el propósito del análisis FODA es "identificar las estrategias para aprovechar las oportunidades externas, contrarrestar las amenazas, acumular y proteger las fortalezas y erradicar las debilidades" (p.18).

Como podemos apreciar, son muchos los factores que intervienen en la planeación de una organización. Generalmente estos factores se dividen en tres etapas: diseño, implementación y evaluación. Para las OSC consideramos que el seguimiento a los programas implementados que tiene que ver con la etapa de evaluación, resulta muy importante para el éxito organizacional.

En este sentido, la OSC debe ser capaz de dar seguimiento a los planes que se hayan realizado, con la intención de realizar una evaluación y corregir los aspectos vulnerables. Además, debido a la naturaleza de las OSC es sumamente importante la realización de alianzas o redes de colaboración, para fortalecer su desempeño y tener un mayor alcance de sus objetivos. 
Podemos concluir, que las OSC, a través de la planeación, serán capaces de implementar diversos programas para alcanzar sus objetivos generales, sin olvidar, en ningún momento, la importancia de la influencia del entorno en el cual se encuentra inserta la organización.

\section{METOdOLOGÍA}

Como se mencionó al principio, la presente investigación está basada en un diagnóstico, el cual consistió en la aplicación de un instrumento en línea con 35 ítems, aplicado a una muestra incidental de 86 OSC. Tanto el diseño del instrumento como la captura y el procesamiento de la información, se llevaron a cabo mediante el servicio en línea limequery. Las variables analizadas se agruparon en 5 ejes estratégicos.

Para este trabajo solamente nos enfocaremos al eje temático relativo a Planeación, en el cual se consideraron 8 preguntas. El objetivo de presentar este eje, es analizar detalladamente la planeación con la que operan las OSC en el estado de Guanajuato, para así, detectar las áreas de mejora y oportunidad en las cuales se pueden implementar nuevas estrategias, y proyectos que mejoren su eficiencia y efectividad, y por ende, su desempeño.

\section{RESULTADOS Y ANÁLISIS}

A continuación, se mencionan los resultados obtenidos de las preguntas realizadas en la encuesta para conocer más acerca de la Planeación Estratégica de las OSC en el estado de Guanajuato.

De acuerdo con la información recopilada de la pregunta 1 que a la letra dice ¿La OSC sistematiza continuamente su experiencia?, el $70 \%$ de las OSC manifiestan que sí sistematizan continuamente su experiencia, es decir, revisan las operaciones y actividades que han realizado para planear lo consecutivo con base en los resultados obtenidos. El 30\% de las organizaciones encuestadas mencionan que no llevan a cabo esta actividad.

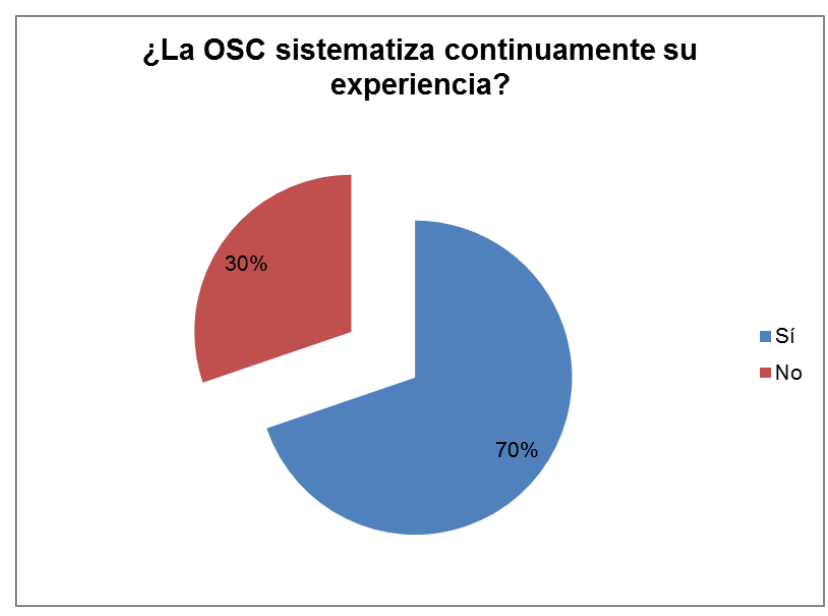

Figura 1. Pregunta 1 - Sistematización de la experiencia

De acuerdo con la figura 1, las organizaciones encuestadas se preocupan por llevar una documentación de todas las actividades que realizan con lo cual se facilita el proceso de planeación y se toman mejores decisiones. Sin embargo, aún se debe trabajar con el $30 \%$ de OSC 
que no sistematizan continuamente su experiencia, lo cual se da por lo demandante de sus actividades. Las causas de lo anterior se pueden deducir dado que generalmente son organizaciones pequeñas, donde los miembros realizan distintas funciones, desde administrar, procurar fondos, atender a beneficiarios, etc.

Los resultados de la pregunta 2 de la encuesta que dice ¿La OSC tiene plasmada en documentos formales su Misión y Visión? señalan que el 92\% de las OSC sí cuentan con misión y con visión; sin embargo, el $8 \%$ manifiesta no tener plasmados estos elementos clave de la planeación.

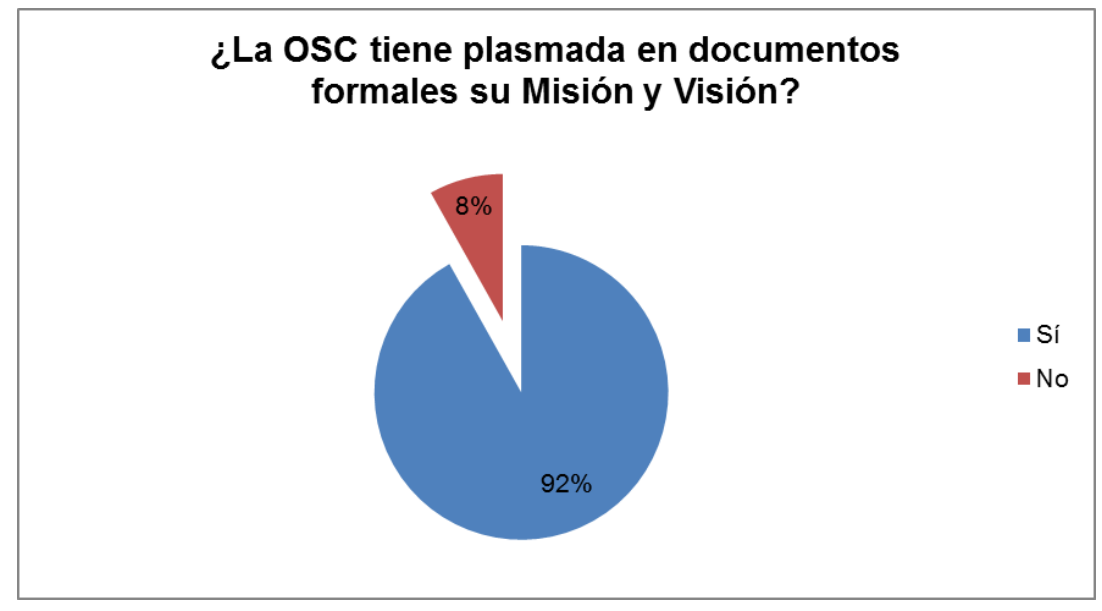

Figura 2. Pregunta 2 - Misión y visión

Con estos datos podemos asumir que las OSC, en su mayoría sí tienen clara su razón de ser y hacia dónde van, pues tienen definidos los elementos clave que las identifican. Resulta revelador que el $8 \%$ restante, menciona que sí los tiene pero no los ha externalizado o escrito en algún documento oficial.

Para la pregunta 3 relacionada con el tipo de documentos que maneja la OSC, se permitió a las organizaciones elegir más de una opción de repuesta, obteniendo así un total de 277 respuestas, y no 86 como en las preguntas anteriores, por esta razón se tiene un promedio de 3.2 respuestas por organización.

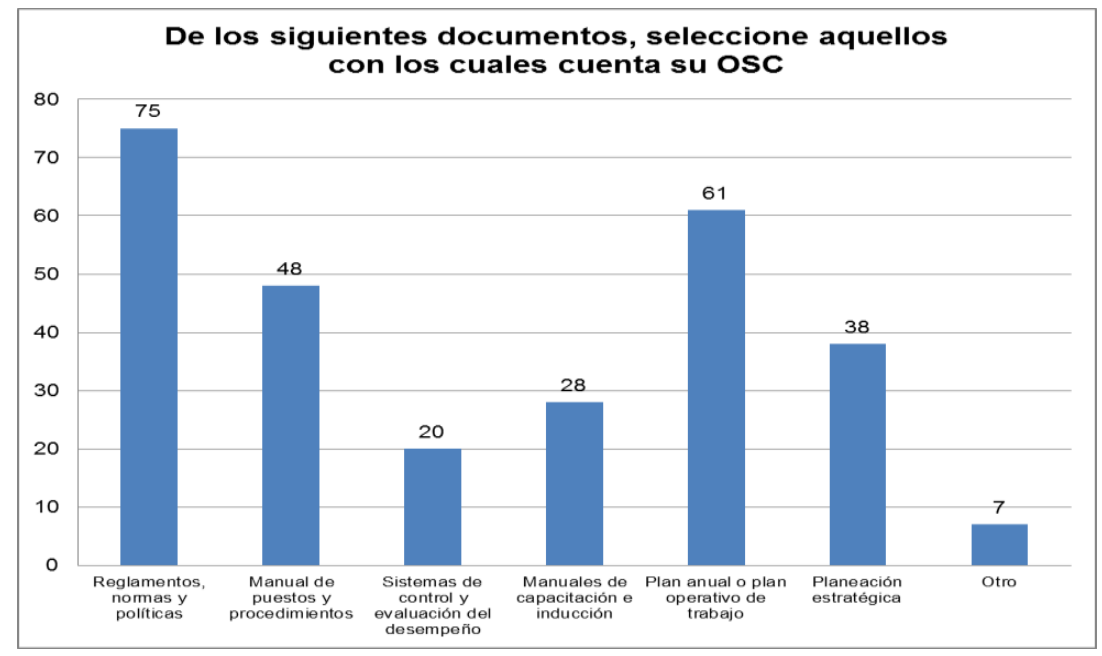

Figura 3. Pregunta 3 - Documentos con los que cuenta la OSC 
De esta manera, el $27 \%$ de las organizaciones encuestadas manifestó contar con reglamentos, normas y políticas; el $22 \%$ con un plan anual o plan operativo de trabajo y el $17 \%$ con manuales de puestos y procedimientos, siendo estos los datos más representativos en los resultados.

En esta pregunta hubo la posibilidad de elegir la opción "otros", así el 3\% de las OSC se inclinó por esta elección y una vez analizadas las respuestas de los encuestados se categorizaron los resultados, dando por resultados las siguientes nuevas opciones: reglamento interno, procedimientos administrativos, código de ética, bitácoras de eventos, certificación ISO 9001:2000 y manuales de capacitación, como se muestra a continuación.

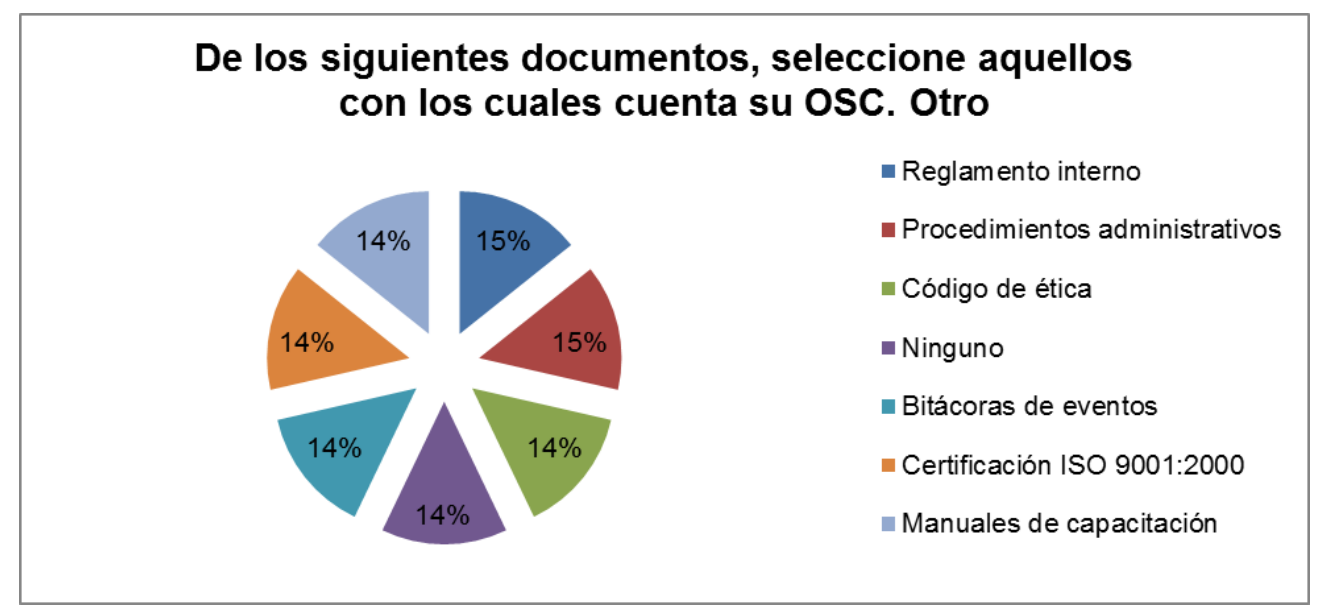

Figura 3. Pregunta 3.1 - Documentos con los que cuenta la OSC. Opción otros

Las respuestas correspondientes a la pregunta 4 respecto a la vinculación y la articulación que tienen las OSC con otras entidades muestran que estas organizaciones se vinculan en su mayoría con el Gobierno Estatal con un 36\%, en segundo lugar con otras OSC (21\%), y en tercer lugar el Gobierno Municipal (19\%); algo que resulta significativo es que el orden de gobierno con el que menos se vinculan es el Federal con un $10 \%$, no obstante ser el que cuenta con más recursos y programas para apoyar a las organizaciones.

Respecto a este ítem, resulta evidente la necesidad que tienen las OSC de incrementar el nivel de colaboración con otros organismos, ya que pueden convertirse en aliados estratégicos tanto para la procuración de fondos, como para la capacitación y la visibilidad.

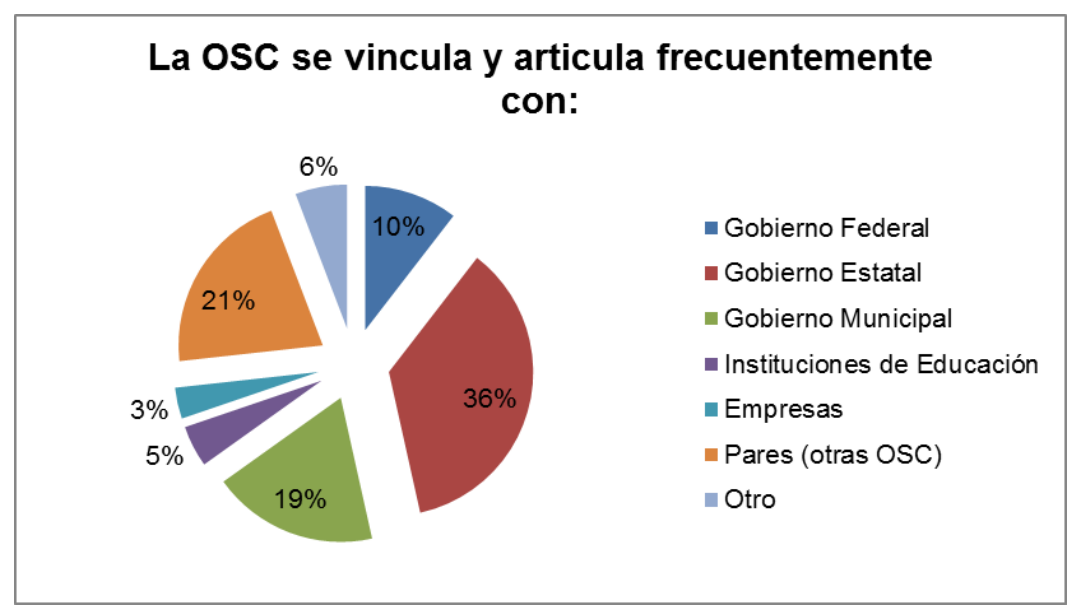

Figura 4. Pregunta 4 - Vinculación y articulación 
Para la pregunta 4 se consideró la opción de "otros", dejando la posibilidad de que los encuestados respondieran libremente. Las opciones alternativas que mencionaron fueron principalmente: la Procuraduría de los Derechos Humanos del estado de Guanajuato y la Comisión Nacional de Derechos Humanos.

Los resultados de la pregunta 5 de la encuesta que a la letra dice ¿La OSC acostumbra hacer diagnósticos acerca de sus fortalezas y debilidades (parte interna) y de sus oportunidades y amenazas (parte externa)? fueron que el $69 \%$ de las OSC manifiestan que sí realizan el diagnóstico FODA, mientras que un $31 \%$ de las OSC encuestas nos respondió que no realizan un análisis FODA.

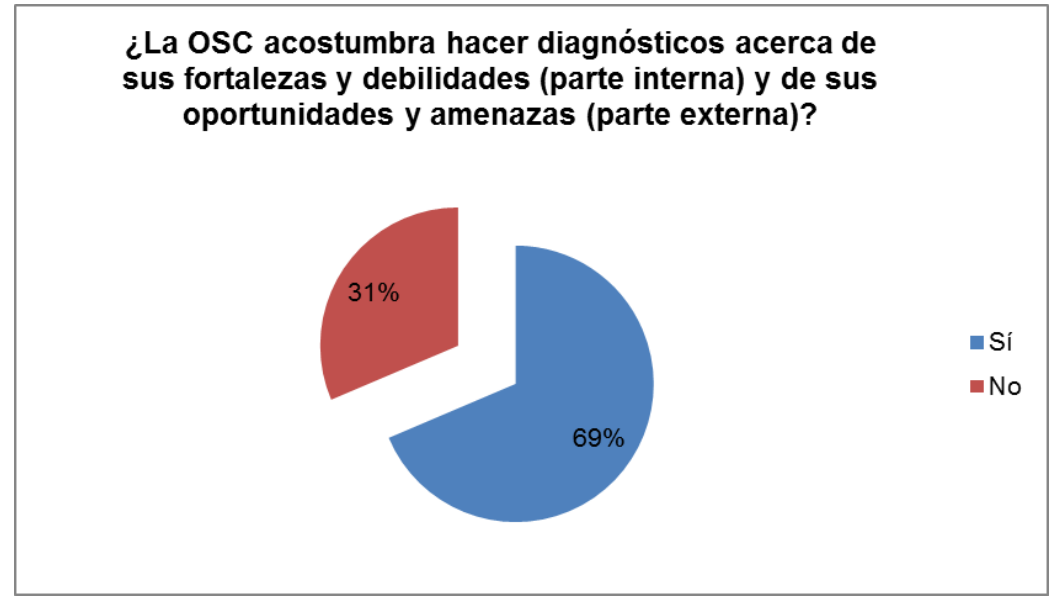

Figura 5. Pregunta 5 - Análisis FODA

Lo anterior da cuenta de que las organizaciones analizadas consideran importante realizar el análisis FODA para saber dónde se encuentran, qué hay que mantener y qué hay que modificar para la adecuada gestión de la organización.

Para la pregunta 6 ¿La OSC es miembro de alguna Agencia o Red de colaboración? los resultados muestran que el $57 \%$ de las OSC que contestaron la encuesta, sí pertenece a alguna agencia o red de colaboración, mientras que el $43 \%$ menciona que no. Como se puede observar, cerca de la mitad de OSC manifiestan no ser miembros de ninguna agencia o red de colaboración, lo cual representa una gran desventaja para ellas.

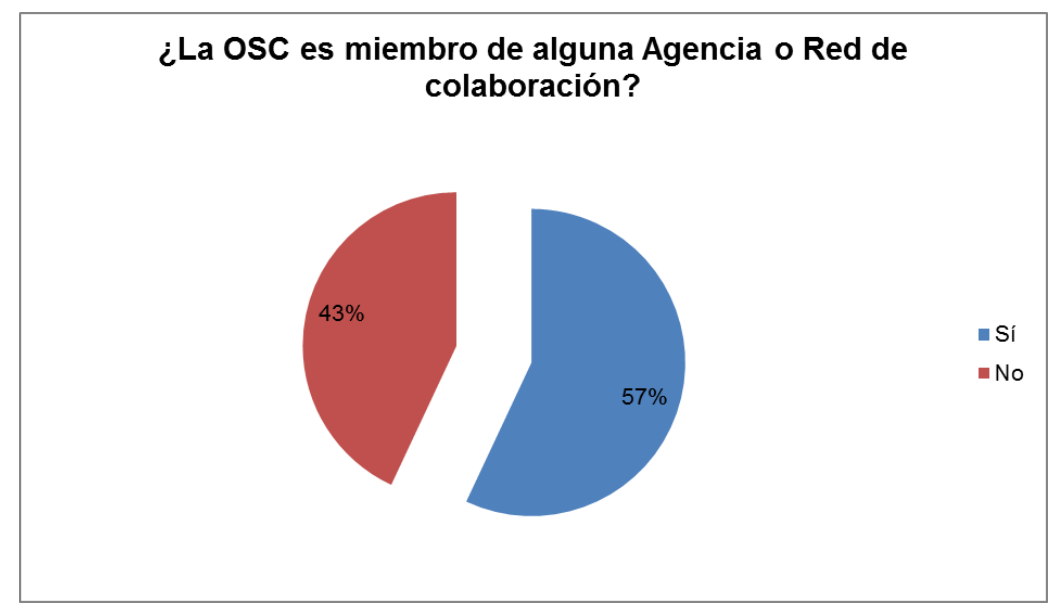

Figura 6. Pregunta 6 - Pertenencia a Agencia o Red de Colaboración 
Para la pregunta 7 ¿La OSC tiene convenios de colaboración firmados con otras instituciones?, el $58 \%$ de la OSC manifiestan que sí tienen convenios firmados con otras instituciones, mientras que el $41 \%$, que representa 36 de las 86 OSC encuestadas, dice no tener ningún tipo de convenios. Podemos asociar esta pregunta con el índice de institucionalidad o formalidad con la cual opera la OSC, ya que puede darse el caso de que la organización sí trabaje con otra OSC, pero de manera informal.

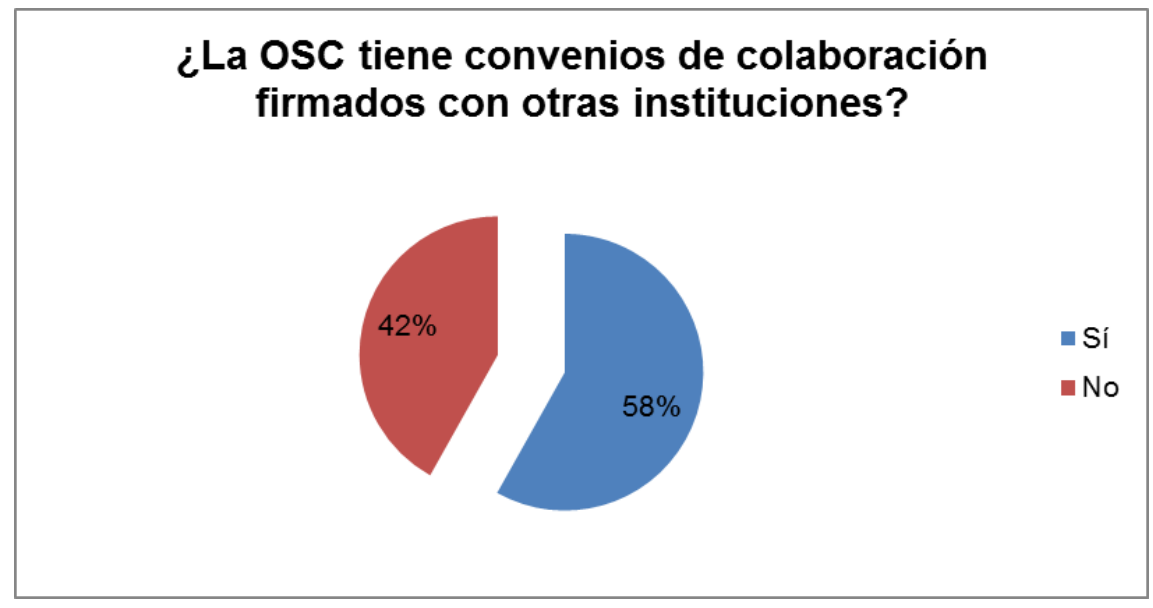

Figura 7. Pregunta 7 - Convenios firmados con otras Instituciones

Finalmente, para la pregunta 8 que dice ¿La OSC da seguimiento a los programas implementados? el resultado de la encuesta arroja que el $73 \%$ de las OSC sí da seguimiento a los programas que implementan, mientras que el $27 \%$ no.

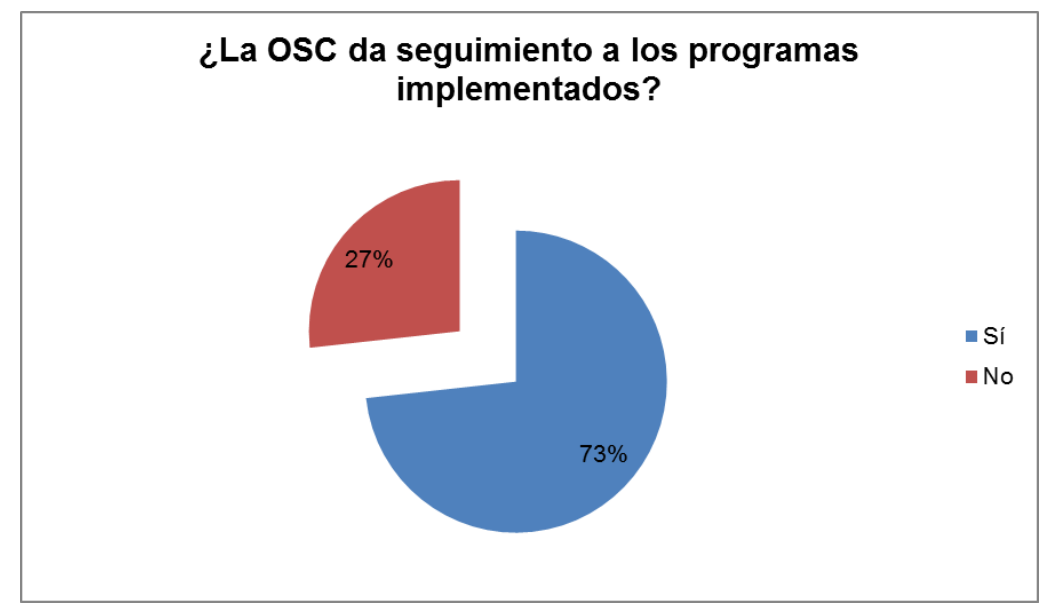

Figura 8. Pregunta 8 - Seguimiento a Programas Implementados

Como se ha mencionado líneas arriba, el dar seguimiento a los programas implementados y su respectiva evaluación, es de gran importancia para cualquier organización, ya que a través de estas actividades se podrá medir el desempeño de la organización, además de que es posible obtener una retroalimentación constante.

La pregunta 8 tuvo una segunda parte referente a los mecanismos empleados para el seguimiento. De acuerdo con los resultados, los mecanismos más recurrentes para dar 
seguimiento a los programas implementados son en primer lugar con $47 \%$ las evaluaciones periódicas (bimestrales, trimestrales, semestrales y anuales), el segundo lugar (27\%) está representado por la evaluación directa con los beneficiarios, mientras que en tercer lugar (12\%) se encuentra el seguimiento a través de presentación de informes a distintas instancias.

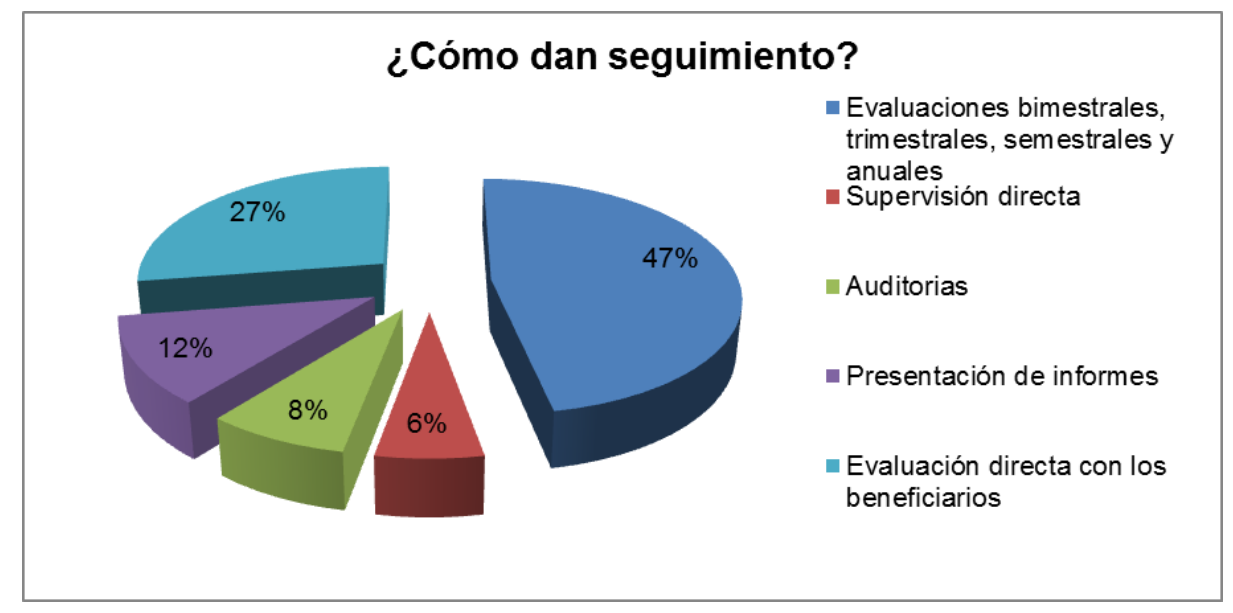

Figura 8.1. Pregunta 8.1 -. Seguimiento a Programas Implementados

Como fue posible apreciar en este breve análisis y con base en las 8 preguntas del diagnóstico aplicado a las OSC del estado de Guanajuato, estas organizaciones sí cumplen con las actividades mínimas de un proceso de planeación estratégica formal, o al menos consideran que sí lo realizan.

Evidentemente tarea pendiente sería conocer los mecanismos reales a través de los cuales las OSC encuestadas realizan la planeación, y cómo es que adquirieron este hábito y expertise.

\section{CONCLUSIONES}

A continuación, se presentan sintéticamente los resultados de la aplicación de la encuesta:

- El $68.97 \%$ de las OSC sistematizan continuamente su experiencia, mientras que el $29.89 \%$ no lo hacen.

- El 91.86 \% de las OSC cuentan con misión y visión, sin embargo el 8.14\% manifiesta no tener plasmados estos elementos clave de la planeación. Estos elementos los plasman en reglamentos, manuales y planes.

- Las OSC se vinculan en su mayoría con el Gobierno Estatal con un $36 \%$, en un segundo lugar con otras OSC (21\%) y en tercer lugar el Gobierno Municipal (19\%), el orden de gobierno con el que menos se vinculan es el federal con un $10 \%$

- El 69\% de OSC manifiestan que sí realizan el diagnóstico FODA, el 31\% no lo realizan.

- $57 \%$ de las OSC pertenece a alguna agencia o red de colaboración, mientras que el $34 \%$ no.

- El $58 \%$ de las OSC tienen convenios firmados con otras instituciones, mientras que el $41 \%$ que representa 36 OSC no tienen.

- El 73\% de las OSC sí dan seguimiento a los programas que implementan, principalmente a través de evaluaciones periódicas. 
Las cifras presentadas pueden darnos una idea general de que la mayoría de OSC lleva a cabo aspectos importantes del proceso administrativo de la planeación. Sin embargo, falta fortalecer aspectos como el hábito de la documentación y sistematización, la vinculación con sectores del gobierno, la participación en redes de colaboración, la evaluación y el seguimiento de las actividades, y la formalización de todo lo anterior.

Con base en esta información y la que hasta el momento se ha recabado de diversas fuentes, a continuación, se mencionan algunas propuestas que consideramos esenciales para que las OSC del estado de Guanajuato fortalezcan su planeación estratégica, en orden de impulsar su desarrollo y sobre todo de alcanzar un mayor impacto social:

- Rumbo claro y compartido, el cual tiene que ver con la esencia y misión de cualquier organización de la sociedad civil, la cual vela por los intereses de una colectividad;

- Adecuada asignación de roles y funciones, lo cual se refiere primordialmente a la tarea de organizar, es decir, saber qué se tiene que hacer y quiénes son los responsables;

- Diseño de indicadores de desempeño, los cuales deben permitir la medición y evaluación constante a partir de metas claras;

- Realización de autodiagnósticos, se trata de estar atentos a lo que sucede tanto en lo interno como en lo externo, es decir, refiere al análisis FODA;

- Colaboración con otros organismos, en general este tipo de organizaciones tiene recursos limitados, por lo cual resulta imprescindible la colaboración y suma de esfuerzos con otras entidades, sean públicas, privada so sociales;

- Articulación y coordinación de la gestión; una adecuada alineación organizacional garantizará el éxito. Se refiere a la concordancia de los planes y programas por área funcional, todos los cuales deben estar orientados al logro de la misión institucional;

- Evaluación, seguimiento y retroalimentación de programas, lo que no se mide, no se puede mejorar, entonces es necesario tener indicadores claros de medición que permita la mejora continua de planes, programas y metodologías de intervención.

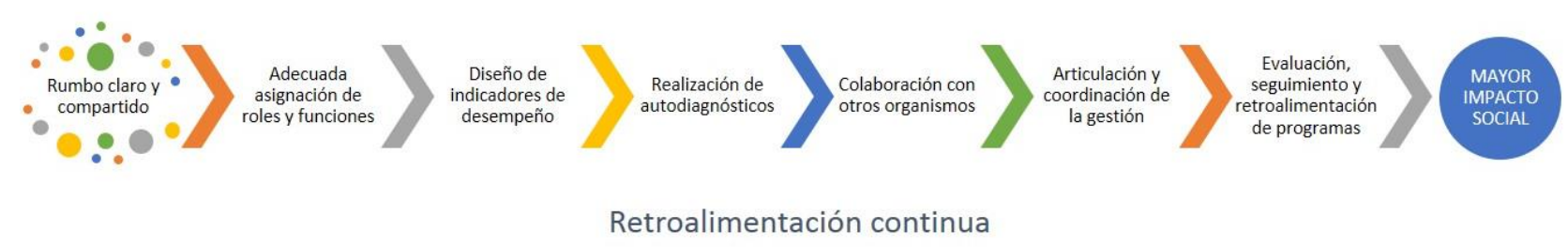

Figura 9. Actividades para reforzar la Planeación Estratégica de las OSC

Siguiendo a Fred (2008) "planear es más que extrapolar el pasado y el presente al futuro. También tiene que ver con desarrollar una misión, pronosticar tendencias y acontecimientos futuros, establecer objetivos y elegir las estrategias que se habrán de seguir" (p. 132); y consideramos que con estas recomendaciones las OSC del estado de Guanajuato estarán en posibilidad de diseñar estrategias que les permitan además de alcanzar sus objetivos, lograr su fortalecimiento.

Se queda en el tintero como futura veta de investigación, el análisis de la evidencia empírica de los procedimientos de planeación, ya que como se mencionó más arriba, solamente se recabó la opinión del líder de la OSC, sin embargo, no se tuvieron medios de verificación de lo manifestado. 


\section{REFERENCIAS}

Britton, B. (2005). Aprendizaje organizacional en ONG. Creando el motivo, los medios y la oportunidad. Cuaderno de Praxis No. 3, USA: INTRAC.

Bryson, J. (2011). Strategic planning for public and nonpofit organizations. 4th edition. USA: Wiley.

Caldera, D. (2013). Organizaciones de la Sociedad Civil y Fortalecimiento. Análisis y propuestas para el estado de Guanajuato. México: Ediciones Fontamara y Universidad de Guanajuato.

Carrillo, P. García, P. y Tapia, M. (2009). El Fortalecimiento Institucional de las OSC en México. Debates, oferta y demanda. México: Alternativas y Capacidades A.C.

Fred, D. (2008). Conceptos de administración estratégica. 11a edición. México: Pearson, Prentice Hall.

Goldfeder, G. \& Aguilar, E. (1997). Planificación y Administración. Un Enfoque Integrador. México: Trillas.

Hill, C. \& Jones, G. (2001). Administración estratégica: Un enfoque integrado. Colombia: McGrawHill.

Hitt, M.A., Ireland, R.D. \& Hoskisson, R.E. (2008). Administración estratégica. Competitividad y globalización. Conceptos y casos. 7ạ edición. México: Cengage Learning.

INEGI [Instituto Nacional de Geografía y Estadística]. (2015). Cuéntame, Número de Habitantes. Disponible en: http://cuentame.inegi.org.mx/poblacion/habitantes.aspx?tema=P. Acceso el 20 de junio de 2016.

Mintzberg, H., Quinn, J., \& Voyer, J. (1993). El Proceso Estratégico: Conceptos, Contextos y Casos. México: Prentice Hall.

RFOSC [Registro Federal de Organizaciones de la Sociedad Civil]. (2016). Buscador de OSC. Disponible en: http://166.78.45.36/portal/. Acceso el 20 de junio de 2016.

Thompson, A., \& Strickland, J. (2004). Administración Estratégica. Textos y Casos, 13a edición. México: Mc Graw Hill.

Wheelen, T., \& Hunger, J. (2008). Administración estratégica y política de negocios, 10a edición, México: Pearson Educación. 